\title{
Index to Volume 17
}

As in previous indexes the subject index is a keyword index of major topics only; writers commented on at some length in discussion articles are indexed but authors of works dealt with in review articles are not included. Numbers refer to the first page of the relevant article. r.a. $=$ review article; disc. $=$ discussion.

\section{Author index}

Bennett, Peter G. 'Modelling Complex Conflicts: Formalism or Expertise?'. 349.

Booth, Ken. 'Security and Emancipation'. 313.

Boucher, David. 'The Character of the History of the Philosophy of International Relations and the Case of Edmund Burke'. 127.

Cohan, A. S. 'Eurotexts and Eurothought: Changing Approaches to the Study of the European Community' [r.a.]. 193.

Cutler, A. Claire. "The "Grotian Tradition" in International Relations'. 41.

Danchev, Alex. 'Life and Death in the South Atlantic' [r.a.]. 305.

Deudney, Daniel; Ikenberry, G. John. 'Soviet Reform and the End of the Cold War: Explaining Large-Scale Historical Change'. 225.

Evans, Tony. 'Human Rights: A Reply to Geoffrey Best' [disc.]. 87.

Farrenkopf, John. 'The Challenge of Spenglerian Pessimism to Ranke and Political Realism', 267.

Groom, A. J. R.; Kostakos, Giorgios; Morphet, Sally; Taylor, Paul. 'Britain and the New UN Agenda: Towards Global Riot Control?' [disc.]. 95.

Heuser, Beatrice. 'NSC 68 and the Soviet Threat: A New Perspective on Western Threat Perception and Policy Making'. 17.

Hollis, Martin; Smith, Steve. 'Beware of Gurus: Structure and Action in International Relations' [disc.]. 393.
Ikenberry, G. John; Deudney, Daniel. 'Soviet Reform and the End of the Cold War: Explaining Large-Scale Historical Change'. 225.

Imber, Mark F. 'Environmental Security: A Task for the UN System' [r.a.]. 201.

Johnson, Edward. 'A Permanent UN force: British Thinking After Suez'. 251.

Johnston, Alexander. 'Weak States and National Security: The Case of South Africa in the Era of Total Strategy'. 149.

Kostakos, Giorgios; Groom, A. J. R.; Morphet, Sally; Taylor, Paul. 'Britain and the New UN Agenda: Towards Global Riot Control?' [disc.]. 95.

Long, David. 'J. A. Hobson and Idealism in International Relations'. 285.

Martin, Laurence. 'Dismantling Deterrence?'. 215. Milner, Helen. 'The Assumption of Anarchy in International Relations Theory: A Critique'. 67. Morphet, Sally; Kostakos, Giorgios; Groom, A. J. R.; Taylor, Paul. 'Britain and the New UN Agenda: Towards Global Riot Control?' [disc.]. 95.

Neumann, Iver B.; Welsh, Jennifer M. 'The Other in European Self-Definition: An Addendum to the Literature on International Society'. 327.

Smith, Steve; Hollis, Martin. 'Beware of Gurus: Structure and Action in International Relations' [disc.]. 393. 
Taylor, Paul; Kostakos, Giorgios; Groom, A. J. R.; Morphet, Sally. 'Britain and the New UN Agenda: Towards Global Riot Control?' [disc.]. 95.

Taylor, Paul. 'The European Community and the State: Assumptions, Theories and Propositions'. 109.

Taylor, Paul. 'The United Nations System under Stress: Financial Pressures and Their Consequences'. 365.

Ward, Veronica. "Regime Norms as "Implicit" Third

\section{Subject index}

Agent-structure problem, 383, 393.

Anarchy, 67.

Antarctica, 167, 201.

Argentina, 167, 305.

Balance of power, 267, 285.

Best, Geoffrey, 87.

Bhaskar, Roy, 393.

Bull, Hedley, 41.

Burke, Edmund, 127.

Capitalism, 225.

Carr, E. H., 215.

Cassin, René, 87.

China, 3.

'Civilization', standard of, 3 .

Cold War, 17, 225, 313.

Consociationalism, 109.

Cuban missile crisis, 349 .

Culture, 327.

Decision-making, 17, 109, 349.

Democratic liberalism, 225.

Dessler, David, 393.

Diplomacy, 285.

Deterrence, 215.

Domestic/international dichotomy, 67.

Drugs, 95.

Elster, Jon, 393

Emancipation, 313.

Environment, 95, 201.

European Community, 109, 193.

Explanation, 225, 383, 393.

Falklands/Malvinas War, 167, 305.

Food and Agriculture Organization (FAO), 365.

Game theory, 167, 349.

Giddens, Anthony, 393.

Globalism, 225.

Gong, Gerrit, 3.
Parties: Explaining the Anglo-Argentine Relationship'. 167.

Welsh, Jennifer; Neumann, Iver B. 'The Other in European Self-Definition: An Addendum to the Literature on International Society'. 327.

Wendt, Alexander. 'Bridging the Theory/Meta-Theory Gap in International Relations' [r.a.]. 383.

Zhang, Yongjin. 'China's Entry into International Society: Beyond the Standard of "Civilization" '. 3 .

Grotius, Hugo, 41.

Historical Reason, 127.

Hobson, J. A., 285.

Holism, 383, 393.

Human rights, 87 .

Idealism, 127, 285 .

Individualism, 383, 393.

Industrial modernism, 225.

Interdependence, 67.

International conflict, 349.

International Labour Organization (ILO), 365.

International law, 87, 167.

International regimes, 41, 167, 201.

International relations theory, 67, 383, 393.

International society, 3; European, 327.

International system, 3, 383.

Knowledge-based questioning systems, 349.

Korean War, 17

Level-of-analysis problem, 383, 393.

Models, 349.

North Atlantic Treaty Organization (NATO), 215

Neo-Marxism, 225.

New Liberal internationalism, 285.

Non-interventionism, 285.

North Sea, 201.

NSC 68, 17.

Other, the, 327.

Ottoman Empire, 327.

Pessimism, 267.

Raison d'état, 327.

Ranke, Leopold von, 267.

Realism, 225, 267, 313; empirical, 127; neo-, 383, 393; scientific, 383, 393; utopian, 313. 
Refugees, 95.

Regime analysis, $41,167$.

Regional integration, 109, 193.

Security, 17, 313; environmental, 201; European, 215; national, 149.

Self-determination, 87 .

South Africa, 149.

Soviet Union, 17, 225.

Sovereignty, 109, 285.

Spengler, Oswald, 267.

State, 109, 149.

Suez crisis, 251.

Terrorism, 95.

Third parties, 167.

Threat perception, 17.
Traditions of international thought, 41, 127.

Turkey, 327.

Understanding, 383, 393.

United Kingdom, 95, 167, 251, 305.

United Nations, 95, 201, 251, 365.

United States, 17, 365.

Universal Declaration of Human Rights, 87.

Universal Moral Order, 127.

Waltz, Kenneth N., 67, 383, 393.

War, 313.

Weak states, 149.

Wendt, Alexander, 393.

Wight, Martin, 41.

World Health Organization (WHO), 365. 